\title{
Editorial
}

\section{Hepcidin and Neutrophil Gelatinase-Associated Lipocalin as a Biomarker for Acute Kidney Injury Linked Iron Metabolism}

Acute kidney injury (AKI) is one of the serious complications of cardiac surgery and can be a significant life-threatening factor associated with high morbidity and mortality [1-3]. There are multiple damaging mechanisms of cardiac surgery-induced $\mathrm{AKI}$, such as ischemia and reperfusion injury, inflammation, and oxidative stress [4]. Among various candidate biomarkers and underlying mechanisms, hepcidin and iron metabolism in AKI are recently attracting the attention of researchers as one of the main mechanisms causing renal damage in various clinical conditions [5-9]. However, the role of iron regulation in cardiac surgery still remains unclear [8].

Hepcidin is mainly synthesized in the liver; however, lower hepcidin expression has also been identified in the kidneys [1, 6]. The main biological role of hepcidin is as an essential regulator of iron metabolism [1, 2, 6]. Hemoglobin, myoglobin, cytochromes, and various enzymes need iron as an important component of their functions [1]. However, excessive iron accumulation and extracellular free-iron can cause tissue injury [1]. Therefore, human body requires delicate and efficient tools to control iron absorption and excretion not only to maximize physiologic iron utility but also to minimize undesirable toxicity [1]. Hepcidin appears to downregulate iron absorption in duodenum and reduce extracellular iron levels [1, 2]. Therefore, hepcidin is known to be a major regulator of iron homeostasis resulting in intracellular iron sequestration [6]; its deficiency induces ironoverload disease such as hereditary hemochromatosis, while its excess results in anemia such as anemia of chronic disease [1].

Besides iron status, two other mechanisms regulate hepcidin levels:erythropoietic activity and systemic inflammation. Hepcidin is downregulated by hypoxia-induced erythropoiesis and upregulated by interleukin (IL)-6 mediated systemic inflammatory reaction $[1,5]$. These multiple mechanisms involving hepcidin metabolism can make clinical interpretation difficult in renal injury.

To avoid injury due to ferroptosis, various iron binding proteins serve as endogenous protective molecules through sequestering labile iron [2, 9]. Neutrophil gelatinase-associated lipocalin (NGAL) is a well evaluated AKI biomarker with a broad spectrum of etiology [3]. Interestingly, NGAL as well as hepcidin are involved in iron metabolism as iron sequestering components $[1,6,9]$. Upregulation of NGAL can be detected in both plasma and urine early after AKI, suggesting possible renal protective mechanism through iron sequestration [2]. Both NGAL and hepcidin show iron sequestering function with different mechanisms [6].

In this issue, Albert, et al. [4] reported a pilot evaluation of various urinary AKI biomarkers linked to iron metabolism and inflammation. They suggested that NGAL, hepcidin, NGAL/hepcidin ratio, and IL-6 were independent predictors of AKI after an open heart surgery [4]. This pilot study may be an interesting precedent for understanding the role of iron metabolism through hepcidin and NGAL in AKI. We expect similar investigations will 
increase both in larger patient groups and in various phenotypes of AKIs, to further elucidate the clinical utility of these biomarkers.

\section{Acknowledgement}

None.

\section{Author contribution}

Both authors equally contributed as a corresponding author.

\section{Conflict of interest}

Nothing to declare.

\section{Research funding}

None.

\section{REFERENCES}

1. Prowle JR, Westerman M, Bellomo R. Urinary hepcidin: an inverse biomarker of acute kidney injury after cardiopulmonary bypass? Curr Opin Crit Care 2010;16:540-4.

2. Scindia Y, Leeds J, Swaminathan S. Iron homeostasis in healthy kidney and its role in acute kidney injury. Semin Nephrol 2019;39:76-84.

3. Kim H, Hur M, Lee S, Marino R, Magrini L, Cardelli P, et al. Proenkephalin, neutrophil gelatinase-associated lipocalin, and estimated glomerular filtration rates in patients with sepsis. Ann Lab Med 2017;37:388-97.

4. Albert C, Haase M, Albert A, Kropf S, Bellomo R, Westphal S, et al. Uri- nary biomarkers may complement the cleveland score for prediction of adverse kidney events after cardiac surgery: a pilot study. Ann Lab Med 2020;40:131-41.

5. Scindia Y, Wlazlo E, Leeds J, Loi V, Ledesma J, Cechova S, et al. Protective role of hepcidin in polymicrobial sepsis and acute kidney injury. Front Pharmacol 2019;10:615.

6. Malyszko J, Bachorzewska-Gajewska H, Malyszko JS, Koc-Zorawska E, Matuszkiewicz-Rowinska J, Dobrzycki S. Hepcidin - potential biomarker of contrast-induced acute kidney injury in patients undergoing percutaneous coronary interventions. Adv Med Sci 2019;64:211-5.

7. Vela D. Systemic and local hepcidin as emerging and important peptides in renal homeostasis and pathology. Biofactors 2019;45:118-34.

8. Choi N, Whitlock R, Klassen J, Zappitelli M, Arora RC, Rigatto C, et al. Early intraoperative iron-binding proteins are associated with acute kidney injury after cardiac surgery. J Thorac Cardiovasc Surg 2019;157: 287-97.

9. Swaminathan S. Iron homeostasis pathways as therapeutic targets in acute kidney injury. Nephron 2018;140:156-9.

Corresponding author: Sun Young Cho, M.D., Ph.D.

(iD https://orcid.org/0000-0002-3208-5446

Department of Laboratory Medicine, School of Medicine, Kyung Hee University Hospital, 23 Kyungheedae-ro, Dongdaemun-gu, Seoul 02447, Korea Tel: +82-2-958-8671, Fax: +82-2-958-8609

E-mail: untoyou@hanmail.net

\section{Co-corresponding author: Mina Hur, M.D., Ph.D.}

(iD https://orcid.org/0000-0002-4429-9978

Department of Laboratory Medicine, Konkuk University School of Medicine, 120-1 Neungdong-ro, Gwangjin-gu, Seoul 05030, Korea

Tel: +82-2-2030-5581, Fax: +82-2-2636-6764

E-mail: dearmina@hanmail.net

Key Words: Hepcidin, Neutrophil gelatinase-associated lipocalin, Urinary biomarker, Acute kidney injury, Iron metabolism, Inflammation 